\title{
Why should syntactic islands exist?
}

\author{
Eran Asoulin
}

(Forthcoming in Mind $\mathcal{E}$ Language)

\begin{abstract}
Sentences that are ungrammatical and yet intelligible are instances of what I call perfectly thinkable thoughts. I argue that the existence of perfectly thinkable thoughts is revealing in regard to the question of why syntactic islands should exist. If language is an instrument of thought as understood in the biolinguistics tradition, then a uniquely human subset of thoughts is generated in narrow syntax, which suggests that island constraints cannot be rooted in narrow syntax alone and thus must reflect interface conditions imposed on the output of the computational system and its mapping to external systems.
\end{abstract}

Keywords: islands; locality; narrow syntax; language as an instrument of thought; perfectly thinkable thoughts.

\section{Introduction}

Natural language requires the computational operation that can build hierarchical structures in a recursive fashion, thus providing the ability to produce and comprehend an unbounded number of sentences. Yet this is not enough: another computational operation is needed that can establish dependencies between positions in the sentence, thus providing information crucial for interpretation. Various dependencies exist between the elements of sentences, so that the relationships between words and phrases is constrained in a particular way. Dependencies include, amongst others, anaphora, case, and wh-dependencies. Whdependencies are an example of long-distance filler-gap dependencies, where the wh-word at the beginning of the sentence can be arbitrarily far away from the theta-role assigning element (a verb in the case of (1) and (2)) later in the sentence that selects the wh-word or phrase as a semantic argument.

(1) What does Jude think that Clayton saw _?

(2) What does Jane think that the audience expects her to perform _ for her recital?

In other words, when the wh-phrase (the filler) is fronted to the beginning of the sentence, it leaves a gap (represented by an underscore above) where speakers of the language expect the argument to be. Like all dependencies, wh-dependencies exemplify the near ubiquitous displacement feature of natural languages in which linguistic elements are pronounced in sentential positions that are different from the positions in which they receive their interpretation. So in (1) the wh-word what must be interpreted as being part of the argument structure of the verb saw, which is its thematic object, but what is pronounced in a position that can be indefinitely far away from the verb. Since wh-dependencies can be arbitrarily far apart (as is evident in (3) and (4) below), they are not constrained by length. 
(3) What does Lilly believe that Jude thinks that Clayton saw _?

(4) What does Peter know that Lilly believes that Jude thinks that Clayton saw _?

However, when we investigate sentences such as (5), (6), and (7), ${ }^{1}$ it becomes clear that longdistance dependencies are constrained in a structural way that has little to do with length or linear order. Words or phrases can be displaced within the sentence, leaving the canonical position empty, but there are certain domains within sentences out of which displacement will result in unacceptable or ungrammatical sentences. Ross (1967) called these domains islands, explaining that the words or phrases are in a sense stuck on that portion of the sentence, for certain syntactic operations cannot cross island boundaries and apply to those words or phrases. The wh-word in (5), (6), and (7) was moved out of an island, but that is not a legitimate grammatical operation and thus the result is an unacceptable sentence.

(5) *What do you wonder [cP whether Jude bought_]?

(6) *What did you make [NP the claim that Jude bought_]?

(7) *What do you think [NP the speech about_] interrupted the broadcast?

The interest in island effects is not merely that they exist, for a complete description of every island effect is just that: a description and not an explanation. The deeper explanatory question is why islands should exist in the first place. Moreover, it is revealing to ask why the particular islands that we observe obtain and not some others. Such questions should be at the heart of any research program seeking to explain why the architecture of the language faculty is the way it is. The investigation of islands is subsumed under the study of locality, which studies the principles that limit the space within which syntactic operations can apply. Locality refers to structural locality, rather than the locality of adjacent words or the relative distance of particular phrases from other phrases in a linear string. As Rizzi (2013) discusses, locality cannot merely be a matter of linear contiguity. Consider agreement in sentences such as This picture of her dear parents pleases Mary, where the verb pleases does not agree with the linearly contiguous noun parents, but rather with the head of the subject noun phrase picture, which is a linearly more distant noun but is "closer" when one considers the hierarchical structure of the sentence. ${ }^{2}$ See (8) below for a simplified illustration.

(8) [s [Np [Np [D This] [n picture]] [pp [p of] [Np [N her] [Adjp [Adj dear] [N parents]]]]] [vp [v pleases] [N Mary]]]

\footnotetext{
${ }^{1}$ Adapted from Sprouse and Hornstein (2013).

${ }^{2}$ See Lasnik and Uriagereka (2012) for more on structural distance.
} 


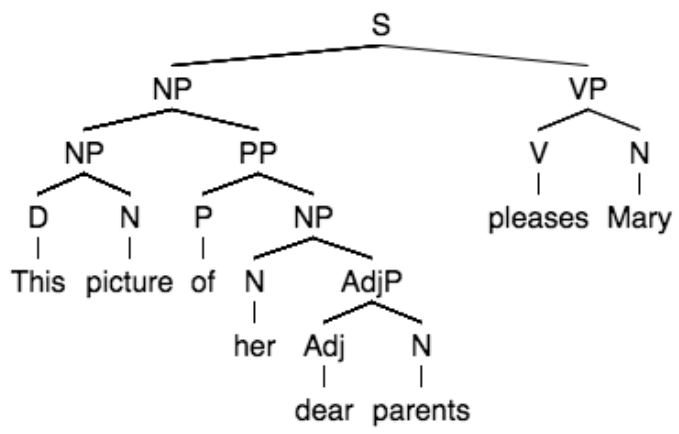

Wh-islands are not by any means the only sort of island effect, for there are many syntactic environments in which long distance dependencies are blocked. Moreover, island phenomena are ubiquitous in every studied language and computational constraints of this sort appear to be universal (see Boeckx 2012; Phillips 2013; 2013a). Consider another example, discussed in Ross (1967: 247ff.), the classic work on islands: in (9) the noun phrase (NP) the boy's cannot be relativised to produce (10).

(9) I disliked the boy's playing the piano loudly.

(10) *The boy whose playing the piano loudly I disliked was a student.

However, the object NP of playing in (9), the piano, can be relativised to produce (11).

(11) The piano which I disliked the boy's playing loudly was badly out of tune.

The explanation of the difference between (10) and (11) is that (10) is a violation of an island constraint and is thus an unacceptable English sentence. Ross (1967: 243) called this the Sentential Subject Constraint, according to which no element dominated by an S may be moved out of that $S$ if that $S$ is dominated by an NP which itself is immediately dominated by S. In other words, clausal subjects are islands and so nothing can be moved out of clausal subjects. So in (9), the NP the boy's cannot be moved because the NP within which it is contained (the boy's playing the piano loudly) is dominated by an S. There is thus an island boundary that bars the movement of the NP the boy's. See (12) for a simplified illustration.

(12) [s [NP I] [vp [v disliked] [NP [s [NP the boy's] [vP [v playing] [NP the piano] [AdvP loudly]]]]]

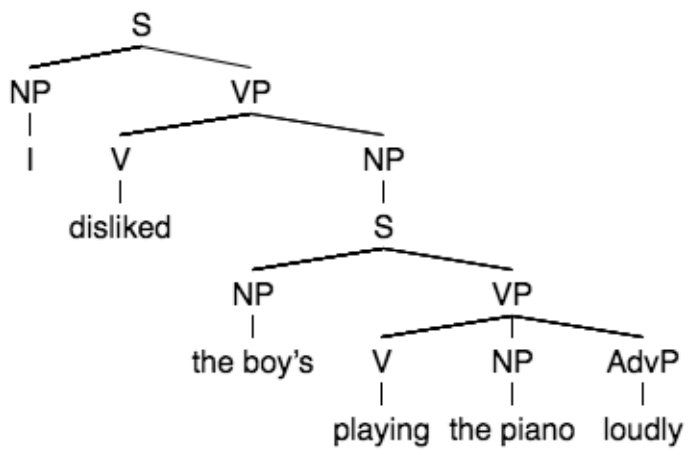


Note that even though (10) is ungrammatical, we can easily understand the sentence, for it is an instance of a perfectly thinkable thought. That is, the content of (10) can be easily grasped and paraphrased but attempting to express it in that way makes it ungrammatical. There are countless cases of island violations of every kind that are easily interpretable but yet are judged to be ungrammatical. Perfectly thinkable thoughts will be discussed further in section 5; for now, they can be understood as the interpretable structures generated by the computational procedures of I-language. I argue below that the existence of perfectly thinkable thoughts suggests that island phenomena are due to interface conditions, as opposed to being constraints on structure generation or due to performance limitations. That is, if language is an instrument of thought as has been argued by various authors in the biolinguistics tradition (Hinzen 2006; 2013; Chomsky 2013; 2013a; Asoulin 2016), then a uniquely human subset of thoughts must be generated in narrow syntax, which suggests that island constraints and the ungrammatical sentences they cause cannot be rooted in narrow syntax alone. This is because speakers must be able to mentally represent thoughts whose expression in sound/sign nevertheless violates island constraints. If islands were due to constraints on structure generation alone then that would leave unexplained perfectly thinkable thoughts. That is, it would leave unexplained why certain structures that have been generated are ungrammatical due to island constraints and yet their meaning is perfectly understandable. If we take interpretable structures to be generated by narrow syntax and then explain their grammaticality or unacceptability in terms of interface relations, then this explains why we are able to interpret sentences with island violations. In other words, the existence of perfectly thinkable thoughts provides independent support for the claim that islands reflect interface conditions imposed on the output of the computational system and its mapping to external systems.

There are, broadly speaking, three types of explanations that have been offered for why islands exist. These are (i) that islands are a result of performance (memory or processing) limitations that constrain how linguistic knowledge is put to use, (ii) that islands reflect certain constraints on structure generation in narrow syntax, and (iii) that islands reflect interface conditions imposed on the output of the computational system and its mapping to external systems. I discuss each in turn in the following three sections. In the final section I suggest that we have independent reasons to believe that islands are best explained in terms of interface conditions. I argue that islands are interface phenomena having to do with the conversion by the sensorimotor interface of hierarchical structures to linear ones. This has to do with the understanding of language as an instrument of thought, specifically that the underlying mechanisms of language make possible a particular kind of thought that is uniquely human. Humans share with animals many kinds of cognitive processes, but there is a uniquely human kind that is rooted in the underlying mechanisms of language. I discuss what implications this has for the explanation of why syntactic islands should exist.

\section{Islands are a result of performance limitations}

Reductionist accounts attempt to explain island phenomena in terms of independently motivated properties of language processing or other cognitive mechanisms. Following Phillips (2013), one should distinguish between such reductionist accounts and what he terms grounded accounts of syntactic phenomena. These two accounts are separate but are too often equivocated. As Boeckx (2012: 27-28) puts it, grounded accounts "do not deny the 
existence of grammatical constraints. What they do is offer a rationale for them in terms of extra-grammatical considerations." That is, "they make roughly the same kind of (synchronic) empirical predictions as standard grammatical accounts." Grounded accounts share with formal accounts the working assumption that syntactic constraints are mentally represented, but grounded accounts have a different explanation for the origin of these constraints. Hawkins (1999), for example, argues that the difficulty in processing filler-gap dependencies is directly reflected in the conventions of grammars. So, on this account, even though syntactic constraints are real and mentally represented, their ultimate source is due to the processing aspects of the parser shaping what the grammars allow or disallow as acceptable structures. ${ }^{3}$ In other words, on the grounded account syntactic constraints are real formal restrictions that reduce language processing difficulty because they arose for that very purpose.

This is not so with reductionist accounts, which take islands to be a matter of performance, not competence. Hofmeister and Sag (2010: 379), for example, argue that once the processing burdens on islands "are properly understood (and explained partly in terms of semantic and pragmatic factors), there remains little work to be done by purely syntactic island constraints." They object to what they see as the consensus in the field according to which, even though the full set of island data cannot be explained in purely syntactic terms, "there remains a strong bias toward treating as much of the data as possible in purely syntactic terms." Performance-based accounts claim that island phenomena are the byproducts of general principles of cognition. The claim is that speakers judge sentences with island effects as unacceptable not because of grammatical constraints but rather because of some overburden on the language processing system. Pritchett (1991) is one of the earliest of such accounts. He argues that island sentences are grammatical but unprocessable. That is, island constraints can be "shown to exceed the capacity of the human sentence processor to perform certain structural analyses during parsing" (Pritchett 1991: 302). Specifically, Pritchett argues that island effects are a type of garden path sentence (e.g., (13) and (14) below) that result from the combination of (a) "local parsing decisions that ultimately prove not to be consonant with a global grammatical representation" and (b) "the parser's inability to perform the structural reanalyses necessary to obtain a grammatical representation" (Pritchett 1991: 304). In other words, the "initial incorrect parsing decision is frequently, but not necessarily, the result of a local ambiguity in the input string", and so garden path sentences (and island effects) "are the result of unrecoverable parsing errors" (Pritchett 1991: 304).

(13) The horse raced past the barn fell.

(14) The doctor persuaded the patient he was having trouble with to leave.

More recently, Hofmeister and colleagues (Hofmeister \& Sag 2010; Hofmeister et al. 2013; building on the work of Deane 1991; Kluender 1992; 1998; Kluender \& Kutas 1993) have argued that factors independently tied to processing difficulty can also affect the acceptability of island constructions. Hofmeister and Sag (2010) discuss psycholinguistic research showing that processing load increases inside a syntactic dependency and also increases as the dependency gets longer. They also discuss separate psycholinguistic

\footnotetext{
${ }^{3}$ For other grounded accounts see Fodor (1974) and Berwick and Weinberg (1984).
} 
research showing that the difficulty of processing filler-gap dependencies is contingent on the properties of the filler itself. So, for example, a "wealth of memory research shows that elaboration or increased semantic processing during the study phase of recall tasks benefits subsequent retrieval of isolated words and propositions" - the retrieval of these words and propositions aids in making the correct syntactic dependencies across phrases and lessens parsing errors. Their claim is that this "memory-based account thus offers one means for explaining why semantically richer, more complex filler phrases promote increased acceptability" (Hofmeister \& Sag 2010: 384). Presumably, then, the more information of this kind the parser has at its disposal, the easier it will be able to process any given sentence. In a way not dissimilar to that of Pritchett (1991), Hofmeister and Sag note that semantically richer filler phrases reduce the chances of garden-pathing. After discussing other factors independently tied to processing difficulty, Hofmeister and Sag present their own experiments that manipulate the aforementioned variables to test whether they have any effect on the acceptability of island constructions. They argue that there are numerous processing pressures that aggregate during sentence comprehension; when the processing difficulty goes beyond a threshold, unacceptability is often the result. They conclude that "the performance-based view accounts for islands as the byproduct of general principles of cognition" (Hofmeister \& Sag 2010: 403-404).

This general claim is qualified in a number of places: for example, they say that "it may well be the case, as already noted, that certain island phenomena are best explained via syntactic constraints, while others may be better and more easily reconciled with processingbased considerations" (Hofmeister \& Sag 2010: 405). They also admit that researchers favouring the formal grammatical explanation of islands accept that some performance factors should play a part in the explanation of island phenomena. If both sides agree that performance and formal factors should play a role, where does that leave the debate? On one side of the debate there is a push to explain as much as possible in terms of formal constraints, and on the other side there is a push to explain as much as possible in terms of processing-based considerations. In regard to the latter, one can of course propose any number of ways in which the processing costs of building long-distance dependencies interact with the processing of island structures, but currently the only theory that contains a mechanism to explain the supposed superadditive effects of processing islands is that of Kluender and Kutas (1993) and variants and elaborations thereof (such as Hofmeister \& Sag 2010). As noted above, this mechanism in effect sets a threshold beyond which the processing system runs out of resource capacity, namely that of working memory.

Sprouse et al. (2012) argue that the main difference between reductionist theories and their competitors lies in the role of working memory capacity. That is, according to the reductionist approach the superadditive effect is due to limits on working memory, whereas on the grammatical account working memory is not part of the explanation. We can thus use working memory, which is the only mechanism proposed to account for the supposed superadditive effects of processing islands, in order to test the empirical predictions of both accounts. Thus, the "reductionist theory would predict a correlation between the size of the limited pool of resources and the size of the resulting island effects. The grammatical theory predicts no such correlation" (Sprouse et al. 2012: 90). Sprouse et al. carried out two studies to test whether the strength of island effects varied across speakers as a function of individual differences in working memory resources. They found no evidence supporting the predictions made by reductionist theories. As they put it, their "results are consistent 
with grammatical theories of island effects, which predict no relation between resource capacity and the strength of island effects" (Sprouse et al. 2012: 118). Moreover, "the known facts of working-memory tasks suggest that the likelihood of finding such a relationship [between island effects and resource capacity] with different tasks or a different sample is extremely small" (Sprouse et al. 2012: 118).

\section{Islands reflect computational constraints}

So the reductionist account remains controversial, and as reductionists themselves readily admit there are certain core island phenomena that are perhaps best explained by syntactic constraints. Let us now look at the syntactic account, which has its roots in Chomsky (1973), according to which islands reflect certain computational constraints on grammatical operations (see Chomsky 2001; 2008; 2013a for recent versions). That is, for computations to be efficient they should apply mostly locally within specific domains; in regard to syntactic computations, these domains correspond to islands. If this is so, then island effects can be explained as the by-products of (presumably language-independent) principles that guarantee efficient computation. In order to understand the nature of the computational constraints that lead to islands, let us briefly sketch the generative procedures and computations inherent in I-languages.

An I-language is the computational system in the mind of individual language users. It is a generative procedure that can output all and only the strings of a particular language. The "I", of course, refers to the internal but also to the intensional nature of particular Ilanguages, meaning that there is a specific procedure encoded in the mind that generates the strings; this is in contrast to the extensional nature of E-language grammars (Chomsky 1986). Another way to put the matter is in terms of formal mathematics, in which a sequence can be defined extensionally by listing its members, say $\{0,1,1,2,3,5,8,13 \ldots\}$, or intensionally by providing a formula that can generate all and only the members of the sequence, say the formula $F_{n}=F_{n-1}+F_{n-2}$ that generates all and only the numbers of the Fibonacci sequence. An intensional definition is much more useful for large sets and is essential for potentially infinite sets like the ones associated with natural languages. The internal computational processes of the language faculty generate linguistic objects that are employed by the conceptual-intentional systems (systems of thought) and the sensorimotor systems. Lexical items, then, and all expressions generated from them, must have properties that are interpretable at both these interfaces. Notice that on this view the language faculty is embedded within, but separate from, the performance systems. So an I-language is a device that generates structured expressions of the form $\operatorname{Exp}=\langle$ Phon, Sem $\rangle$, where Phon provides the sound instructions of which the sensorimotor systems make use, and Sem provides the meaning instructions of which the systems of thought make use.

The expression Exp is generated by the operation Merge, which takes objects already constructed and constructs from them a new object. So, for example, Merge $(X, Y)$ will yield the unordered set $\{X, Y\}$. The structure-building operation Merge follows the principle of Minimal Computation (compute and articulate as little as possible), for it is the simplest possible computational operation for the task at hand (Berwick \& Chomsky 2016; Chomsky 2016). There are two cases of Merge: External Merge refers to the operation where two syntactic objects are Merged but where neither one is part of the other. Internal Merge, on the other hand, refers to the operation where one of the syntactic objects is part of the other. For example, Internal Merge takes place when a syntactic object is combined with the set 
that contains it: so if Merge $(X, Y)$ yields $Z=\{X, Y\}$, then Merge $(Z, X)$ yields $\{X, Y\{X\}\}$. For concreteness, take the following simplified example of External Merge. The silver saucer broke yesterday is produced by Merge as follows: lexical items are Merged to (separately) create The, silver, and saucer. Then silver and saucer are Merged to create the Noun Phrase (NP) silver saucer. Then silver saucer is Merged with the to create the NP the silver saucer. Then that NP is combined with the Verb Phrase (VP) broke yesterday (which was produced by Merge when lexical items were Merged to create broke and lexical items were Merged to create yesterday, and then broke and yesterday were Merged together to create the VP broke yesterday).

As for Internal Merge, suppose we Merged which saucer with John broke which saucer to produce which saucer John broke which saucer, which via further computations is then externalised as which saucer did John break. Before externalisation, there are two copies of the same linguistic object: the original one and the displaced one. They are both essential for interpretation. As Chomsky has remarked in various places when he discusses Merge, this is an example of the ubiquitous phenomenon of displacement in language, where phrases are heard in only one place but are interpreted both there and in another place. So we interpret the above sentence to mean "for which $X$, John broke the saucer X". Merge, then, defined as recursive set-formation, produces hierarchical structures and allows for the unbounded embedding of these structures (namely, it allows for discrete infinity).

What are Merged are of course not words but rather their properties, namely their syntactic features. It is features that are the building blocks of syntax and the way they combine and agree with each other shapes the way lexical items and phrases are structured. An important distinction in regard to features is that between interpretable features, which have an effect on semantic interpretation, and uninterpretable features, which make no difference to the semantics of a sentence but are required for its syntax. In order for the semantic interface rules to apply, the syntactic operations must create a structure that has no uninterpretable features. This means that in the course of a syntactic derivation all uninterpretable features need to be eliminated, for if they are not then the derivation crashes and a complete semantic interpretation is not possible. As Adger and Svenonius (2011) discuss, the notion of feature matching is the driving force behind the establishment of syntactic dependencies. The presence of uninterpretable features forces feature matching and when this occurs each uninterpretable feature is deleted as it is matched with an interpretable feature.

Let us look at a simplified concrete example of feature checking adapted from Adger (2003). Suppose we want to generate the VP kiss pigs. The word kiss $[\mathrm{V}, u \mathrm{~N}]$ has an interpretable feature [V], which is needed for its semantic interpretation as an event, and an uninterpretable feature $[u \mathrm{~N}]$, which is needed for the syntax to determine the category of syntactic elements which will be able to Merge with kiss. In this case the feature determines that kiss can Merge with a lexical item that has $[\mathrm{N}]$ as one of its features. The word pig[N] has the interpretable feature $[\mathrm{N}]$ and can thus Merge with kiss. When kiss $[\mathrm{V}, u \mathrm{~N}]$ is Merged with pigs $[\mathrm{N}]$ to create the $\mathrm{VP}$, the interpretable feature $[\mathrm{N}]$ is checked against the uninterpretable feature $[u \mathrm{~N}]$ and the latter is deleted. This derivational system determines the grammaticality of sentences based on whether a well-formed derivation can be constructed for them: this is done by feature checking. When the derivation crashes (i.e., when some uninterpretable features were not checked) an ungrammatical sentence results. Let us now look at how the derivational approach explains islands.

Consider the following two sentences, again adapted from Adger (2003). 
(15) I believed [DP the claim [CP that Philip would invade the city of Athens]].

(16) *Which city do you believe [DP the claim [CP that Philip would invade]]?

The reason why (16) is ungrammatical is that the complementiser phrase (CP) and determiner phrase (DP) are phases, which means that once constructed anything within these two categories is inaccessible for feature matching later in the derivation. Syntactic derivations are assumed to take place in cyclic steps called phases, and the Phase Impenetrability Condition stipulates that once a phase is complete any syntactic object within it is unavailable for further feature checking (Chomsky 2008). Thus, in (16) the uninterpretable wh-feature cannot match with the wh-expression and so the derivation crashes leading to an island effect. This is because the DP is a phase and the Phase Impenetrability Condition means that the syntactic objects within it are unavailable for further feature matching.

\section{Islands reflect conditions on the output of the computational system}

There are two ways in which formal constraints can cause island phenomena. The first was discussed in the previous section, which took constraints to apply to structure generation. What follows from this first view is that "the constraint makes it impossible for speakers to construct mental representations that violate the constraint" (Phillips 2013: 158). An alternative, but still formal, account takes the constraints to "apply as a filter on generated structures, meaning that speakers are able to mentally represent structures that violate the constraint, but that they somehow also encode the fact that those representations are grammatically illicit" (Phillips 2013: 158, emphasis in original). Boeckx (2012) makes the distinction between pure syntax approaches and interface approaches. The latter, "if they are to gain some traction (explanatory force), must appeal to properties of the external systems with which syntax interacts, and determine which of these properties lead to certain syntactic outputs to be judged illicit" (Boeckx 2012: 56).

The pure syntax approach is problematic in light of what is known as island repair, where island structures do not lead to unacceptability when certain conditions are met. Ross (1967; 1969) showed early on that if certain conditions are met then islands do not necessarily lead to unacceptability. One type of island repair that has been extensively studied is what Ross (1969) termed "sluicing", which is an ellipsis phenomenon in which the sentential part of a constituent question is elided, resulting in only the wh-phrase remaining. Compare (17) to (18), which are taken from Ross (1969: 252).

(17) Ralph is going to invite somebody from Kankakee to the party, but they don't know who he's going to invite to the party.

(18) Ralph is going to invite somebody from Kankakee to the party, but they don't know who.

As the contrast between (19) and (20) below shows, the wh-movement in sluicing is insensitive to islands. In other words, the relative clause island in (19) is repaired or circumvented by sluicing in (20) (see Merchant 2008 from which (19) and (20) are taken). 
(19) *They want to hire someone who speaks a Balkan language, but I don't remember which they do.

(20) They want to hire someone who speaks a Balkan language, but I don't remember which.

Another type of island repair, also discussed by Ross, is resumption, where a resumptive pronoun is inserted where we would otherwise expect a gap to be: this resumptive pronoun repairs or circumvents the island, or at least makes the sentence more acceptable. Compare (21) and (22) below, taken from Asudeh (2004: 320).

(21) *I'd like to meet the linguist that Peter knows a psychologist that works for _.

(22) I'd like to meet the linguist that Peter knows a psychologist that works for her.

Boeckx (2012: 79) remarks that island repair poses a problem not only for derivational approaches "but also for those accounts like Uriagereka's [1999] that let interface considerations influence the course of syntactic derivations, for the two types of accounts are designed to prevent the island-violating extraction from taking place." It is, says Boeckx, "a very serious puzzle for such approaches if it indeed turns out that the relevant extraction can be shown to have taken place, only to be legitimized by a subsequent, post-syntactic operation. How can something be repaired if it was never constructed in the first place? How can something be legitimized in certain contexts if it is predicted never to happen in any context?" There are two related problems here: one is that island repair implies that movement from island domains can be done but that a subsequent operation like sluicing or resumption somehow hides this from the external systems interfacing with narrow syntax (thus allowing a sentence like (21) to be judged unacceptable). The other problem is that if movement from island domains cannot be done, so that no movement dependency can be established across a relative clause island as in (19), then a subsequent operation like sluicing appears to hide this fact from the external systems (thus allowing (20) to be judged as an acceptable sentence).

It appears that a narrow syntax explanation of islands is problematic in light of island repair, at least if it is understood as the view that takes constraints to apply to structure generation alone. Returning to the aforementioned remark from Phillips (2013: 158), what follows from the narrow syntax explanation of islands is that "the constraint makes it impossible for speakers to construct mental representations that violate the constraint." However, island repair phenomena and perfectly thinkable thoughts are evidence that speakers can construct mental representations that violate island constraints. This is because many island sentences, whether unacceptable or repaired, are intelligible. In other words, as has been known for a long time, there is a disconnect between a speaker treating a structure as interpretable and treating that same structure as grammatically wellformed. This suggests that island structures are generated in narrow syntax and are assigned an interpretation and that the unacceptable nature of island sentences arises from the interface with the sensory-motor systems. In other words, narrow syntax generates perfectly thinkable thoughts, but when the latter are externalised via sound/sign then communication problems like island phenomena appear.

Strictly speaking, then, one could say that there are no island phenomena at Sem because the generated structures are interpretable there but are uninterpretable or cause problems at Phon. "Bad" or unacceptable sentences are only "bad" at Phon, they cause no 
problems at Sem. Notice that this is not the same as saying that since a sentence with an island is unacceptable but comprehensible, that implies that a "bad" or problematic sentence syntax was generated (so it's not the case that a "bad" sentence was generated and then "fixed" by island repair). I argue that there is no problem with the sentence's syntax, for the thought it expresses via Sem is perfectly intelligible. The only problem that arises is when that thought is externalised in acts of communication. This is an important distinction. The structure is fine for internal thought but causes problems for communication. I flesh out this argument in the next section. But first, let us see the details of the view that island constraints apply as an interface filter on generated structures.

Recall that under the derivational account, islands are explained as a result of successive cyclic movement, as a result of the way in which syntactic derivations are computed in successive phases. The derivations that crash are ungrammatical or unacceptable but are "often used as literary devices and in informal discourse, with a precise and felicitous interpretation at the interfaces" (Chomsky 2008: 144). In other words, according to the derivational account, the derivation that has crashed is still transferred to the Sem and Phon interfaces where each interface must still assign the crashed derivation an interpretation. According to the interface account I outline below, however, narrow syntax allows the island-violating movement to occur (and hence the derivation does not crash) and so islands are due to operations that take place mostly, if not entirely, at the interfaces. It follows from the principle of Minimal Computation that "as soon as the information is transferred [to the interfaces] it will be forgotten, not accessed in subsequent stages of derivation: the computation will not have to look back at earlier phases as it proceeds, and cyclicity is preserved in a very strong sense" (Chomsky 2008: 143). This is the aforementioned Phase Impenetrability Condition (PIC) which Boeckx (2012: 60-61) argues is both too strong and too weak. It is too strong because in light of island repair is does not offer any escape hatch, and it is too weak because "once an escape hatch is provided, it becomes necessary to provide a restrictive theory of when this escape hatch can be exploited, for, otherwise, all opacifying (read: island-inducing) effects of the PIC can be voided. Unfortunately, to this day, no such theory is available."

The solution offered by Boeckx, which is of the kind I favour, is still very much a formal account and keeps most aspects of the narrow syntax account. The difference is in what is taken to follow from the principle of Minimal Computation. Boeckx (2012: 120, emphasis mine) explains:

[...] islands are inevitable side-effects of a grammatical system that is designed in such a way as to ensure, via cyclicity, an efficient computation - one that seeks to reduce computational bottlenecks such as multiple roots as soon as they arise. A side-effect of immediately spelling out certain structures is that certain elements within these see their fates being sealed "too early." Although nothing in syntax prevents these elements from moving, the external systems that receive early information about them take that information to be complete, and set these elements aside, effectively turning them into islands.

The way in which narrow syntax computes interpretable structures and the subsequent form in which these structures are then transferred to the interfaces at Spell-Out are the source of island phenomena. Notice that on this account narrow syntax freely generates perfectly 
thinkable thoughts without recourse for externalisation and then interface operations at Phon determine whether the form of externalisation is acceptable or not in regard to islands. Boeckx believes that the problem with islands could be present at both interfaces. However, the presence of perfectly thinkable thoughts along with the language as an instrument of thought hypothesis offer convincing evidence that the problem is mostly, if not entirely, at the Phon interface. Let us look at a specific proposal to this end.

A key property at the Phon interface that creates islands is the need to convert hierarchical structures, which have no linear order, to structures that are linear and thus appropriate for externalisation. Kayne's (1994) influential account, according to which linear order is part of Phon and is derived from asymmetrical c-command relations, was the first of several versions of an interface explanation of islands (see also Uriagereka 1999; 2011; Fox \& Pesetsky 2005; Hornstein et al. 2007). According to Uriagereka's (1999; 2011) interface account, complex syntactic units need to be linearised early on in the derivation and then returned to the syntax as if they were single, atomic lexical items. The claim is that this early Spell-Out and linearisation process leads to islands because once Spell-Out takes place the relevant units are unavailable for further computation. The linearisation process involves multiple applications of Spell-Out at Phon and turns successive parts of the derivation into islands. Hornstein et al. (2007) is another recent version of this so-called dynamic account.

For concreteness, let us consider a simplified derivation, taken from Fox and Pesetsky (2005: 10ff.). According to their account, when a domain is spelled out the linearisation instructions are added to an "ordering table". Each new Spell-Out cycle adds new ordering information to the ordering table, which gives Phon information about the order of pronunciation. Suppose $\alpha$ merges with D to yield (23).

(23) $\alpha[\mathrm{D}$ X Y Z]

Spell-Out gives the ordering instructions to Phon as $X$ precedes $Y$, and $Y$ precedes $Z(X<Y$, $\mathrm{Y}<\mathrm{Z}) .{ }^{4}$ Now suppose that $\mathrm{X}$ undergoes leftward movement into a higher Spell-Out domain $\mathrm{D}^{\prime}$ to yield (24).

(24) $\left[\mathrm{D}^{\prime} \ldots \mathrm{X} \alpha\left[\mathrm{D} t_{\mathrm{x}} \mathrm{Y} Z \mathrm{Z}\right]\right]$

The linearisation of $\mathrm{D}^{\prime}$ now adds further ordering information to the ordering table. So in addition to $\mathrm{X}<\mathrm{Y}$ and $\mathrm{Y}<\mathrm{Z}$ we now also have $\mathrm{X}<\alpha$ and $\alpha<\mathrm{D}$ (meaning that $\alpha<\mathrm{Y}$ ). So the instructions in the ordering table are now: $\mathrm{X}<\mathrm{Y}, \mathrm{Y}<\mathrm{Z}, \mathrm{X}<\alpha, \alpha<\mathrm{Y}$. Notice that the ordering statements generated when $\mathrm{D}$ was spelled out are consistent with the ordering statements added when $\mathrm{D}^{\prime}$ was subsequently spelled out.

Consider now an alternative scenario in which $\mathrm{Y}$ (instead of $\mathrm{X}$ ) moves leftwards:

$(25) *\left[D^{\prime} \ldots Y \propto\left[D X t_{\mathrm{y}} \mathrm{Z}\right]\right]$

The instructions in the ordering table in this alternative scenario are: $\mathrm{X}<\mathrm{Y}, \mathrm{Y}<\mathrm{Z}, \mathrm{Y}<\alpha, \alpha<\mathrm{X}$. There is now a contradiction, for the original ordering statements generated when $\mathrm{D}$ was spelled out are not consistent with the new ordering statements added when Y moved

${ }^{4}$ I follow Fox and Pesetsky in using "<" to mean "precedes". 
leftward. This is because the first Spell-Out instructed Phon to pronounce X before $Y$, but the instructions at the subsequent Spell-Out instructed Phon to pronounce $Y$ before $X$. That is, if $\alpha$ must be pronounced before $\mathrm{X}$, and if $\mathrm{X}$ must be pronounced before $\mathrm{Y}$, how can $\mathrm{Y}$ be pronounced before $\alpha$ ? Islands are the result of such contradictions between sets of phonological instructions in the ordering table. This is because once information about linearisation is established at the end of a given Spell-Out domain (and sent to Phon as instructions for pronunciation), it cannot be deleted or altered in the course of subsequent Spell-Out operations.

Notice that this dynamic account (as well as that of Uriagereka et al.) sees the need for linearisation at the Phon interface as influencing the course of the syntactic derivation, for the aim is to prevent island-violating movement from taking place. So in regard to (23), for example, Fox and Pesetsky argue that $Y$ may not move leftwards out of $D$ if it phonologically crosses $\mathrm{X}$. There is thus a direct connection established between islands and the timing of Spell-Out. Boeckx (2012), on the other hand, argues that there is no strong theoretical reason to ban movement out of islands. Indeed, I think that we have good reason to suppose that movement is allowed, for if island structures are interpretable (and given that language is an instrument of thought) the structures must have been generated in narrow syntax.

Another way to put the matter is as follows. There is nothing in the syntax that produces problematic structures such as islands. Rather, the problems only appear when the structures are sent to Phon for linearisation and externalisation. This is compatible with language being an instrument of thought because the way in which structures are generated by narrow syntax are perfectly interpretable at Sem but cause problems at Phon. Islands cannot be due to constraints on structure generation at narrow syntax because if this was the case then narrow syntax could not supply Sem a structure rich enough for interpretation that islands clearly make available. ${ }^{5}$ I argue that we can keep the account where islands are due to a contradiction of phonological instructions at Spell-Out and also hold that movement is allowed out of islands. The latter explains why we can interpret island sentences, and the former explains why we find them unacceptable.

The aim of minimalism is to examine "every device (principle, idea, etc.) that is employed in characterizing languages to determine to what extent it can be eliminated in favor of a principled account in terms of general conditions of computational efficiency and the interface condition that the organ must satisfy for it to function at all" (Chomsky 2004: 106). Therefore, any account that reduces islands to interface conditions as much as possible is to be preferred. The dynamic account retains elements of the derivational account in that constraints from the external systems influence structure generation. But the account I suggest goes further by claiming that islands are mostly, if not entirely, a product of externalisation at Phon, leaving narrow syntax to freely generate structures that are perfectly interpretable at Sem. In other words, in order to be usable at all by the sensorimotor systems, the structures generated by narrow syntax must be linearised, for telepathy and speaking in structures are of course unavailable. If islands could be shown to be a by-product of linearisation, leaving narrow syntax to generate structures freely, that would go a long way to satisfying the aims of the minimalist program. In addition, if it could be shown that

\footnotetext{
${ }^{5}$ I thank an anonymous reviewer for pointing out the need to flesh out this part of my argument and for helping me put the matter in these terms.
} 
perfectly thinkable thoughts are interpretable for reasons having to do with computational efficiency at the Sem interface, then that would strengthen the case for island phenomena being due to constraints that exist, perhaps entirely, at the interface at Phon. I suggest in the next section how this can be done. I argue that the biolinguistic understanding of language as an instrument of thought offers an independent reason in support of the interface account.

\section{The asymmetry of the interfaces}

As mentioned above, the structure-building operation Merge follows the principle of Minimal Computation, for it is the simplest possible computational operation for the task at hand. There are independent reasons to believe that cognitive processes satisfy the principle of Minimal Computation (Cherniak 1994; Cherniak et al. 2002; Chomsky 2016), and since Merge satisfies this principle and is able to account for the underlying mechanisms of language, we have strong grounds for its existence as a core computational principle of human language. We saw above that islands cannot be due to constraints on structure generation alone, for they are perfectly thinkable thoughts, and if that is the case then that gives weight to the interface account of islands. We have structures that create problems for communication but that are necessary for interpretation at Sem. There is thus an asymmetry between the interfaces in favour of the semantic side, pushing externalisation via Phon to the periphery.

Another way to put the matter is as follows. The Sem interface is practically uniform among languages, but the Phon interface is of course different for each language. That is, the structures generated at Sem are the same for all languages, for all humans are able to think the same kinds of thought, but those structures are externalised via Phon in different ways. The design of language, then, favours minimal computation at Sem, often at the expense of ease of communication (Sigurðsson 2004; Burton-Roberts 2011; Asoulin 2016). If one takes the biolinguistic understanding of language as an instrument of thought then the existence of islands follows from the normal application of the generative procedures of the language faculty. The problem with island structures only arises when the structures are externalised; these structures are not problematic for internal thoughts. ${ }^{6}$ The upshot is that the structure and content of lexical and phrasal meanings are composed independently of how or whether they are to be communicated.

There is a suite of arguments that support the claim that language is an instrument of thought (Hinzen 2006; 2013; Burton-Roberts 2011; Chomsky 2013; 2013a; Asoulin 2016), let us now see what the claim amounts to in detail. Linguistic expressions provide a perspective (in the form of a conceptual structure) on the world, for it is only via language that certain perspectives are available to us and to our thought processes. This is in line with a long rationalist tradition in the philosophy of language and linguistics (Chomsky 1966), most famously articulated by Humboldt in the nineteenth century, according to which language provides humans with a Weltansicht or worldview that allows us to form the concepts with

\footnotetext{
${ }^{6}$ This goes a long way to explain the cross-linguistic variation in island phenomena. Underlying all languages is the same generative procedure that produces perfectly thinkable thoughts at Sem, but these thoughts are externalised in different ways in different languages via Phon. So the same thought externalised in one language might cause islands but that same thought externalised in another language will not. The Phon in each language is different but the Sem is the same. Though note that cross-linguistic variation in islands is quite limited (see Phillips 2013: 9ff for discussion and references).
} 
which we think certain kinds of thought (but, crucially, not all kinds of thought: for we share many kinds of thought processes with animals that do not have language).

In his study of Humboldt, Underhill (2009) remarks that Humboldt's "rich and dynamic model of language" is one "in which the individual both shapes and is shaped by the organ of speech" (Underhill 2009: xi, emphasis in original). The worldview concept of Weltansicht, which forms the cornerstone of Humboldt's linguistic philosophy, is understood as "the configuration of concepts which allow conceptual thought" of a certain kind (Underhill 2009: 56). Language is an instrument of thought in this sense, but note that this is not a Whorfian claim of linguistic determinism, for thought is certainly independent of language, and what can be expressed or thought by a speaker of one language can certainly be expressed or thought by a speaker of a very different language. As Underhill (2009: 57) remarks, Whorfian claims are merely "weak echoes of Humboldt's voice". Language provides us with a unique way of thinking and talking about the world that is unavailable to non-linguistic animals. Though of course animals have thoughts of many kinds (many of which are shared with humans), but since they lack the language faculty there is a specific kind of thought that they lack (Rogers \& Hauser 2010; Hinzen 2013; Asoulin 2019). Let us see how this rationalist understanding of the role of language in cognition is manifested in current biolinguistics.

Any theory of Sem must satisfy three basic conditions of adequacy, so that in order to capture what the language faculty determines about the meaning of an expression, Sem must "be universal, in that any thought expressible in a human language is representable in it; an interface, in that these representations have an interpretation in terms of other systems of the mind/brain involved in thought, referring, planning, and so on; and uniform" (Chomsky 1995: 21). Sem must be uniform "for all languages, so as to capture all and only the properties of the system of language as such" (Chomsky 1995: 21). Note the stress on all and only the properties of the system of language: the language faculty allows humans to use available concepts (some of which are shared with other animals) to introduce formally new concepts. The claim is not that Sem is the interface of all conceptual content or of all of thought.

Pietroski $(2008 ; 2010 ; 2018)$ has developed one of the most interesting and detailed accounts of this internalist semantics, the leading idea of which is that "in the course of language acquisition, humans use available concepts to introduce formally new concepts that can be fetched via lexical items and combined via certain operations that are invoked by the human faculty of language" (Pietroski 2010: 247, emphasis in original). That is, meanings are (internal, and unconscious) instructions for how to access and assemble concepts of a special sort. Meaning is here understood not in an extensional sense but rather in terms of the cognitive resources (the computational procedures) that humans deploy in generating the meanings. To put the matter in Pietroski's terms, already existing concepts (many of which we share with other animals) are lexicalised and in the process distinctively new concepts are produced that we are then able to combine to form linguistic expressions. Just as the formally new concepts are not a mere concatenation of their constituents, the linguistic expressions generated are also distinctively new in a way that is not a mere concatenation of their constituent concepts. This process of lexicalisation and structure-building, the product of which are perfectly thinkable thoughts, is unique to humans and is part of the explanation of the creative aspect of language use (Chomsky 1966; McGilvray 2001; 2005; Asoulin 2013). 
In order to flesh out the idea that lexicalisation is the process by which pre-existing concepts are used to introduce formally new concepts, Pietroski moves away from the Fregean idea that combining expressions is an instruction to saturate a concept and towards a Conjunctivist account of linguistic composition (Hornstein \& Pietroski 2009; Pietroski 2018). According to the latter account, lexicalisation is not a process in which a previously available concept is merely labelled using a lexical item that inherits its content from the concept itself. Rather, lexicalisation is a device for accessing previously available concepts which become lexical items that are used as input to I-language operations that combine the lexical items in specific ways to introduce new formally distinct concepts. Accordingly, the Sem of any expression Exp $=\langle$ Phon, Sem $\rangle$ is not a concept that is paired with a pronunciation. Indeed, as Pietroski puts it, "evaluating SEMs as if they were concepts may be a category mistake, like evaluating an instruction to fetch a rabbit as male or female" (Pietroski 2010: 252, emphasis in original). So a Sem is an instruction to fetch (i.e., lexicalise) a previously available concept that is then used to build a formally new concept(s). This formally new concept will be stored in the mind somehow (and perhaps be recombined with other concepts to create yet more formally new concepts), but the Sem itself is not a concept.

Another way to put the matter is as follows. Humans possess a great variety of prelexical mental representations (many of which we share with other animals). On the Conjunctivist account, these pre-lexical mental representations are linked to formally distinct but analytically similar concepts. The latter are sometimes referred to as I-concepts (Jackendoff 1989; 1990) to signal that the way in which these concepts are to be studied is on the model of the study of language signalled by the use of I-language as opposed to Elanguage. Thus, "the repertoire of I-concepts expressed by sentences cannot be mentally encoded as a list, but must be characterized in terms of a finite set of mental primitives and a finite set of principles of mental combination that collectively describe the set of possible Iconcepts expressed by sentences" (Jackendoff 1990: 9). I-concepts, then, generate a uniquely human subset of concepts that humans can use to think about the world. The internalist semantics claim is that understanding an expression of I-language (or perceiving its meaning) is a matter of (unconsciously) recognising that that expression is an instruction to construct concepts of a special kind. This explanation of meaning also offers an explanation for the creative aspect of language use, for it sheds light on the computational procedure by which we combine concepts in recursively productive ways to yield formally new concepts. Notice again that this biolinguistic understanding of meaning does not focus on externalisation. The interpretable structures are generated in a way that is optimal at Sem but that is often problematic at Phon.

To recap, what follows from the above understanding of language as an instrument of thought in regard to islands? What can we conclude from the understanding of language as providing humans with a Weltansicht or worldview that allows us to form the concepts with which we think certain kinds of thought? The unacceptable island structures that are interpretable must have been generated in narrow syntax (for they are perfectly thinkable thoughts), and this suggests that island phenomena are not due to constraints on structure generation but rather due to the mapping of perfectly thinkable thoughts to the Phon interface. That is, the attempt to externalise perfectly thinkable thoughts leads to communication problems and depending on the I-language of the speaker/hearer the externalisation might lead to island phenomena. 


\section{References}

Adger, David. 2003. Core syntax: A minimalist approach. Oxford: Oxford University Press.

Adger, David \& Peter Svenonius. 2011. Features in minimalist syntax. In Cedric Boeckx (ed.), The Oxford Handbook of linguistic minimalism, 27-51. Oxford: Oxford University Press.

Asoulin, Eran. 2013. The creative aspect of language use and the implications for linguistic science. Biolinguistics 7. 228-248.

Asoulin, Eran. 2016. Language as an instrument of thought. Glossa: a journal of general linguistics 1(1): 46. 1-23.

Asoulin, Eran. 2019. Phrase structure grammars as indicative of uniquely human thoughts. Language Sciences 74. 98-109.

Asudeh, Ash. 2004. Resumption as resource management. Palo Alto: Stanford University. Doctoral dissertation.

Berwick, Robert C. \& Amy S. Weinberg. 1984. The grammatical basis of linguistic performance. Cambridge, MA: MIT Press.

Berwick, Robert C. \& Noam Chomsky. 2016. Why only us: Language and evolution. Cambridge, MA: MIT Press.

Boeckx, Cedric. 2012. Syntactic islands. Cambridge: Cambridge University Press.

Burton-Roberts, Noel. 2011. On the grounding of syntax and the role of phonology in human cognition. Lingua 121. 2089-2102.

Cherniak, Christopher. 1994. Philosophy and computational neuroanatomy. Philosophical Studies 73. 89-107.

Cherniak, Christopher, Zekeria Mokhtarzada \& Uri Nodelman. 2002. Optimal-wiring models of neuroanatomy. In Giorgio A. Ascoli (ed.), Computational neuroanatomy: Principles and methods, 71-82. Totowa, New Jersey: Humana Press.

Chomsky, Noam. 1966. Cartesian linguistics: A chapter in the history of rationalist thought. New York: Harper \& Row.

Chomsky, Noam. 1973. Conditions on transformations. In Stephen R. Anderson \& Paul Kiparsky (eds.), A Festschrift for Morris Halle, 232-286. New York: Holt, Rinehart, \& Winston.

Chomsky, Noam. 1986. Knowledge of language: Its nature, origin, and use. New York: Praeger Publishers.

Chomsky, Noam. 1995. The minimalist program. Cambridge, MA: MIT Press.

Chomsky, Noam. 2001. Derivation by phase. In Michael Kenstowicz (ed.), Ken Hale: A life in language, 1-52. Cambridge, MA: MIT Press.

Chomsky, Noam. 2004. Beyond explanatory adequacy. In Andriana Belletti (ed.), Structures and beyond: The cartography of syntactic structures, Volume 3, 104-131. Oxford: Oxford University Press.

Chomsky, Noam. 2008. On phases. In Robert Freidin, Carlos P. Otero \& Maria Luisa Zubizarreta (eds.), Foundational issues in linguistic theory: Essays in honor of Jean-Roger Vergnaud, 133-166. Cambridge, MA: MIT Press.

Chomsky, Noam. 2013. What kind of creatures are we? The Journal of Philosophy 90(12). 645700 .

Chomsky, Noam. 2013a. Problems of projection. Lingua 130. 33-49.

Chomsky, Noam. 2016. Minimal computation and the architecture of language. The Tsuru University Graduate School Review 20. 7-17. 
Deane, Paul. 1991. Limits to attention: A cognitive theory of island phenomena. Cognitive Linguistics 2. 1-63.

Erteschik-Shir, Nomi. 1992. Resumptive pronouns in islands. In Helen Goodluck \& Michael Rochemont (eds.), Island constraints: Theory, acquisition and processing, 89-108. Dordrecht: Springer-Science+Business Media, B.V.

Fodor, Janet D. 1978. Parsing strategies and constraints on transformations. Linguistic Inquiry 9. 427-473.

Fox, Danny \& David Pesetsky. 2005. Cyclic linearization of syntactic structure. Theoretical Linguistics 31. 1-45.

Hawkins, John A. 1999. Processing complexity and filler-gap dependencies across languages. Language 75. 224-285.

Hinzen, Wolfram. 2006. Mind design and minimal syntax. Oxford: Oxford University Press.

Hinzen, Wolfram. 2013. Narrow syntax and the language of thought. Philosophical Psychology 26(1). 1-23.

Hofmeister, Philip \& Ivan A. Sag. 2010. Cognitive constraints and island effects. Language 86. 366-415.

Hofmeister, Philip, T. Florian Jaeger, Inbal Arnon, Ivan A. Sag \& Neal Snider. 2013. The source ambiguity problem: Distinguishing the effects of grammar and processing on acceptability judgments. Language and Cognitive Processes 28(1-2). 48-87.

Hornstein, Norbert, Howard Lasnik \& Juan Uriagereka. 2007. The dynamics of islands: Speculations on the locality of movement. Linguistic Analysis 33. 149-175.

Hornstein, Norbert \& Paul M. Pietroski. 2009. Basic operations: Minimal syntax-semantics. Catalan Journal of Linguistics 8, 113-139.

Jackendoff, Ray. 1989. What is a concept, that a person may grasp it? Mind \& Language 4(1), 68-102.

Jackendoff, Ray. 1990. Semantic structures. Cambridge, MA: MIT Press.

Kayne, Richard S. 1994. The antisymmetry of syntax. Cambridge, MA: MIT Press.

Kluender, Robert. 1992. Deriving islands constraints from principles of predication. In Helen Goodluck \& Michael Rochemont (eds.), Island constraints: Theory, acquisition and processing, 223-58. Dordrecht: Kluwer.

Kluender, Robert. 1998. On the distinction between strong and weak islands: A processing perspective. In Peter Culicover \& Louise McNally (eds.), Syntax and semantics, vol. 29: The limits of syntax, 241-79. San Diego, CA: Academic Press.

Kluender, Robert, \& Marta Kutas. 1993. Subjacency as a processing phenomenon. Language and Cognitive Processes 8. 573-633.

Lasnik, Howard \& Juan Uriagereka. 2012. Structure. In Ruth Kempson, Tim Fernando \& Nicholas Asher (eds.), Handbook of the philosophy of science. Volume 14: Philosophy of linguistics, 33-61. Amsterdam: North Holland, Elsevier.

McGilvray, James. 2001. Chomsky on the creative aspect of language use and its implications for lexical semantics studies. In Federica Busa \& Pierrette Bouillon (eds.), The language of word meaning, pp. 5-27. Cambridge: Cambridge University Press.

McGilvray, James. 2005. Meaning and creativity. In James McGilvray (ed.), The Cambridge Companion to Chomsky, pp. 204-222. Cambridge: Cambridge University Press.

Merchant, Jason. 2008. Variable island repair under ellipsis. In Kyle Johnson (ed.), Topics in ellipsis, 132-153. Cambridge: Cambridge University Press. 
Phillips, Colin. 2013. Some arguments and nonarguments for reductionist accounts of syntactic phenomena. Language and Cognitive Processes 28(1-2). 156-187.

Phillips, Colin. 2013a. On the nature of island constraints. I: Language processing and reductionist accounts. In Jon Sprouse \& Norbert Hornstein (eds.), Experimental syntax and island effects, 64-108. Cambridge: Cambridge University Press.

Pietroski, Paul M. 2008. Minimalist meaning, internalist interpretation. Biolinguistics 2(4), 317-341.

Pietroski, Paul M. 2010. Concepts, meanings and truth: First nature, second nature and hard work. Mind \& Language 25(3), 274-278.

Pietroski, Paul M. 2018. Conjoining meanings: Semantics without truth values. Oxford: Oxford University Press.

Pritchett, Bradley L. 1991. Subjacency in a principle-based parser. In Robert C. Berwick, Steven P. Abney \& Carol Tenny (eds.), Principle-based Parsing: Computation and Psycholinguistics, 301-345. Dordrecht: Kluwer.

Rizzi, Luigi. 2013. Locality. Lingua 130. 169-186

Rogers, James \& Marc D. Hauser. 2010. The use of formal language theory in studies of artificial language learning: A proposal for distinguishing the differences between human and nonhuman animal learners. In Harry van der Hulst (ed.), Recursion and human language, 213-231. Berlin: De Gruyter Mouton.

Ross, John Robert. 1967. Constraints on variables in syntax. Cambridge, MA: Massachusetts Institute of Technology. Doctoral dissertation. [Published in 1986 as Infinite syntax! Norwood, NJ: Ablex.]

Ross, John Robert. 1969. Guess who? In Robert I. Binnick, Alice Davison, Georgia M. Green \& Jerry L. Morgan (eds.), Papers from the Fifth Regional Meeting of the Chicago Linguistic Society, 252-286. Chicago: University of Chicago.

Sigurðsson, Halldór Ármann. 2004. Meaningful silence, meaningless sounds. Linguistic Variation Handbook 4(1), 235-259.

Sprouse, Jon, Matthew W. Wagers, \& Colin Phillips. 2012. A test of the relation between working-memory capacity and syntactic island effects. Language 88(1). 82-123.

Sprouse, Jon \& Norbert Hornstein. 2013. Experimental syntax and island effects: Toward a comprehensive theory of islands. In Jon Sprouse \& Norbert Hornstein (eds.), Experimental syntax and island effects, 1-20. Cambridge: Cambridge University Press.

Sprouse, Jon, Matthew W. Wagers, \& Colin Phillips. 2013. Deriving competing predictions from grammatical approaches and reductionist approaches to island effects. In Jon Sprouse \& Norbert Hornstein (eds.), Experimental syntax and island effects, 21-41. Cambridge: Cambridge University Press.

Underhill, James W. 2009. Humboldt, worldview and language. Edinburgh: Edinburgh University Press.

Uriagereka, Juan. 1999. Multiple Spell-Out. In Samuel D. Epstein \& Norbert Hornstein (eds.), Working minimalism, 251-282. Cambridge, MA: MIT Press.

Uriagereka, Juan. 2011. Spell-Out and the Minimalist Program. Oxford: Oxford University Press. 